\title{
Thermodynamic magnon recoil for domain wall motion
}

\author{
Peng Yan, ${ }^{1}$ Yunshan Cao, ${ }^{2}$ and Jairo Sinova ${ }^{1}$ \\ ${ }^{1}$ Institut für Physik, Johannes Gutenberg Universität Mainz, Staudinger Weg 7, 55128 Mainz, Germany \\ ${ }^{2}$ Kavli Institute of NanoScience, Delft University of Technology, Lorentzweg 1, 2628 CJ Delft, The Netherlands \\ (Received 10 April 2015; revised manuscript received 13 September 2015; published 30 September 2015)
}

\begin{abstract}
We predict a thermodynamic magnon recoil effect for domain wall motions in the presence of temperature gradients. All current thermodynamic theories assert that a magnetic domain wall must move toward the hotter side, based on equilibrium thermodynamic arguments. Microscopic calculations, on the other hand, show that a domain wall can move either along or against the direction of heat currents, depending on how strong the magnonic heat currents are reflected by the domain wall. We have resolved the inconsistency between these two approaches by augmenting the theory in the presence of thermal gradients by incorporating in the free energy of domain walls a heat current term present in nonequilibrium steady states. The condition to observe a domain wall propagation toward the colder regime is derived analytically and can be tested by future experiments.
\end{abstract}

Spincaloritronics is the subfield of spintronics which explores spin-dependent phenomena coupled to thermal gradients [1]. A very important question within this field is how a magnetic domain wall (DW) can move under a temperature gradient. This question has attracted much attention owing to its applicability in magnetic insulators [2-6] for potential applications in logic devices [7] and data storage technology [8]. The conventional approach using static magnetic fields [9] is well established with high DW velocities [10-12], but does not allow for the synchronous motion of multiple domain walls. Synchronous current-induced domain wall motions due to spin-transfer torques [13-15] and/or spin-orbit torques [16-20] give an alternative way to efficiently manipulate the magnetization configuration, but the required high-current densities cause problems, such as Joule heating owing to Ohmic losses. Heat itself has been proposed as an efficient control parameter to overcome the problems during the emergence of spincaloritronics [21]. Fully understanding and predicting new controlled ways to move the domain walls by magnonic heat currents is paramount to exploiting all their future device possibilities.

There is at present a theoretically incomplete understanding of the temperature-gradient-driven DW motion. There are two types of theories, i.e., a macroscopically thermodynamic theory [2-6] and a microscopically magnonic one [22-24]. The theories contradict each other in certain regimes. In previous thermodynamic theories [2-4], a magnetic domain wall at finite temperature $T$ is treated as a thermodynamic object with free energy, $F=U-T S$, where $U$ is its internal energy which generally can consist of exchange energy, magnetic anisotropy, dipolar interaction, Dzyaloshiskii-Moriya coupling, etc., and $S$ is the entropy. The free energy of the DW can also be expressed as the difference between a system with a DW minus that of the same system without the wall $\Delta F=$ $\Delta U-T \Delta S$. Thermodynamic calculations [2-4] show that, far below the Curie temperature, the entropy $\Delta S(T)$ increases and the free energy $\Delta F(T)$ decreases with the temperature. This leads to a conclusion that the DW must move towards regions with higher temperatures due to the entropic force, with the propagation velocity proportional to the temperature gradient $\nabla T[2-5]$. This tendency has been observed in experiments [6].
On the other hand, there are microscopic angular momentum transfer [22] and linear momentum transfer [23] theories for the magnon-driven DW motion. The DW moves along the opposite direction of current flows if the angular momentum transfer mechanism dominates $[5,22]$, while along the same direction when the linear momentum transfer is more important [23-25], i.e., there are strong spin-wave reflections by the wall $[23,26]$. The proposed mechanism of magnonic linear momentum transfer has been confirmed in various systems including ferromagnets [23,24], antiferromagnets [27,28], and spin textures with Dzyaloshiskii-Moriya interaction [29].

In this Rapid Communication, we show that the inconsistency between these two types of theories can be resolved by augmenting the free energy of the equilibrium thermodynamic theory by a term from the heat current which always flows in a nonequilibrium steady state in the presence of a temperature gradient. The heat current is modified by the DW with momentum-conserving backscatterings. It then leads to a force that pushes a DW toward the colder region. We predict a new thermodynamic magnon recoil effect for the domain wall motion in temperature gradients. Under conditions of a strong backscattering, a high magnon thermal conductivity, and a slow magnon group velocity, this magnon recoil effect surpasses the previously identified entropic force [2-5]. Such a regime can be achieved in yttrium iron garnet (YIG) and other ferromagnetic insulators, as we show below.

We consider a one-dimensional (1D) magnetic wire connecting two thermal reservoirs with temperatures $T_{h}$ and $T_{l}\left(T_{h}>T_{l}\right)$ as shown in Fig. 1 (local thermal reservoirs to define local temperatures are not shown). The temperature gradient $\nabla T$ then drives magnon heat currents $\mathbf{j}_{\varepsilon}^{\mathrm{I}}$ and $\mathbf{j}_{\varepsilon}^{\mathrm{II}}$ in a uniformly magnetized wire (as shown in the upper panel in Fig. 1) and in a wire with a domain wall (shown in the lower panel in Fig. 1), respectively, at nonequilibrium steady states. Dynamics of a ferromagnet at finite temperature (far below the Curie temperature) can be described by a stochastic Landau-Lifshitz-Gilbert equation [30,31]

$$
\frac{\partial \mathbf{m}}{\partial t}=-\mathbf{m} \times\left(\mathbf{h}+\mathbf{h}_{\mathrm{fl}}\right)+\alpha \mathbf{m} \times \frac{\partial \mathbf{m}}{\partial t},
$$

where $\mathbf{m}$ is the unit vector in the direction of local magnetization textures, $\alpha$ is the Gilbert damping constant 


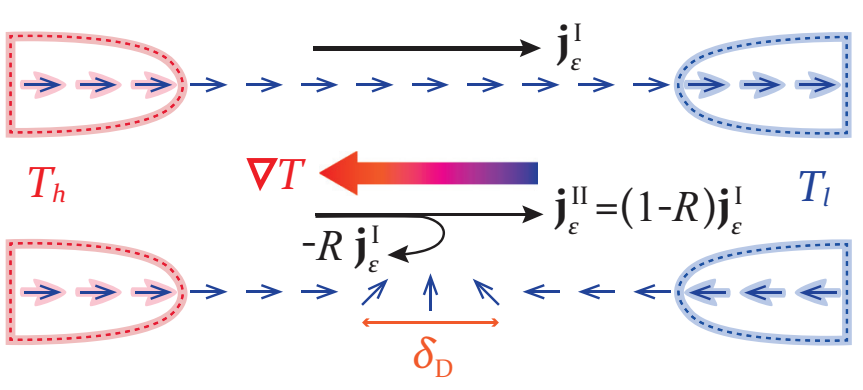

FIG. 1. (Color online) Heat currents in a 1D magnetic wire in the presence of a temperature gradient $\nabla T$ without (upper panel) and with (lower panel) a domain wall with width $\delta_{D}$, denoted by $\mathbf{j}_{\varepsilon}^{\mathrm{I}}$ and $\mathbf{j}_{s}^{\mathrm{II}}$, respectively. The presence of the domain wall causes the reflection of heat currents with a probability $R$.

[31], $\mathbf{h}=-\delta U / \delta \mathbf{m}$ is the effective field conjugate to $\mathbf{m}$, and $\mathbf{h}_{\mathrm{fl}}$ is the fluctuating field due to heat bath (phonon excitations at local equilibrium). Brown [30] formulated a relation between the random field and the damping term through a fluctuation-dissipation theorem $[32,33]$ based on equilibrium thermodynamics (with no temperature gradients). Our present system, however, is in nonequilibrium steady states in the presence of temperature gradients. To the best of our knowledge, a general fluctuation-dissipation theorem for magnetic nonequilibrium steady states does not exist, and the derivation is beyond the scope of present paper. We therefore postpone the discussion of stochastic field $\mathbf{h}_{\mathrm{fl}}$ for future work and only focus on the deterministic magnetization dynamics. Before invoking a fluctuation-dissipation theorem in nonequilibrium, the thermomagnonic torque always predicts a domain wall motion along the temperature gradient, while the Brownian thermophoresis may give the opposite effect.

Luttinger [34] proposed a "gravitational" potential approach to describe the deterministic effect of the temperature gradient, by considering the coupling between the energy density of the system and the scalar potential with no microscopic origin addressed. Tatara [35] revisited this issue by proposing a thermal vector potential which couples to the heat current of the system, in order to avoid the inconsistency of Luttinger's approach. A heat current $\mathbf{j}_{\varepsilon}^{\mathrm{I} \text {,II }}$ can modify the rate of the change of the entropy via a simple relation $[35,36]$

$$
\frac{d S^{\mathrm{I}, \mathrm{II}}}{d t}=-\int d x \mathbf{j}_{\varepsilon}^{\mathrm{I}, \mathrm{II}} \cdot \frac{\nabla T^{\mathrm{I}, \mathrm{II}}}{\left(T^{\mathrm{I}, \mathrm{II}}\right)^{2}} .
$$

This entropy change modifies the free energy [35]. One thus can always write the free energy of the wall as

$$
\Delta F=\Delta F_{\mathrm{e}}+\Delta F_{\text {ne }} .
$$

Here $\Delta F_{\mathrm{e}}=k_{B} T \int \ln \left(1-e^{-\beta \varepsilon}\right) \Delta \rho(\varepsilon) d \varepsilon$ is the equilibrium (time-independent) part with Boltzmann constant $k_{B}, \beta=$ $1 /\left(k_{B} T\right)$, and magnon energy $\varepsilon$, derived by Ref. [4]. It may originate from both a temperature-dependent exchange field included in $\varepsilon$ [2,3] and the difference of magnon density of state (DOS) $\Delta \rho(\varepsilon)$ between a DW and domain [4]. The presence of a domain wall also leads to a constant internal-energy change $\Delta U$ which does not affect any of our arguments. While $\Delta F_{\text {ne }}$ is the nonequilibrium (time-dependent) part proportional to the heat current, with change rate

$$
\begin{aligned}
\frac{d\left(\Delta F_{\mathrm{ne}}\right)}{d t} & =\int\left(T^{\mathrm{I}} \frac{d S^{\mathrm{I}}}{d t}-T^{\mathrm{II}} \frac{d S^{\mathrm{II}}}{d t}\right) d x \\
& =R(T)\left|\mathbf{j}_{\varepsilon}^{\mathrm{I}}\right| \ln \left(\frac{T_{h}}{T_{l}}\right) \geqslant 0,
\end{aligned}
$$

where $R(T)=1-\left|\mathbf{j}_{\varepsilon}^{\mathrm{II}}\right| /\left|\mathbf{j}_{\varepsilon}^{\mathrm{I}}\right|$ denotes a temperature-dependent reflection probability of magnon heat currents by the wall, and $\mathbf{j}_{\varepsilon}^{\mathrm{I}}=-\kappa \nabla T$ is the heat current in the wire without a wall. Here $\kappa$ is the positive-definite magnon thermal conductivity. The temperature dependence of the free energy $\Delta F(T)=$ $\Delta F_{\mathrm{e}}+\Delta F_{\text {ne }}$ is crucial to drive the DW propagation. It has been shown that the equilibrium part $\Delta F_{\mathrm{e}}$ decreases with an increasing temperature $T$ [2-4], which leads to a conclusion that the DW must move to the hotter region to reduce the free energy due to the entropic force. Following the same procedure in Refs. [3,4] to solve Eq. (1) without random fields, one can obtain the corresponding effective field that acts on the wall as $\mathbf{h}_{\mathrm{e}}=\nabla T\left(J_{0} / T_{c}\right) /\left(\delta_{D} m_{\mathrm{eq}} M_{s} a\right)$ with nearest-neighbor exchange energy $J_{0}$, Curie temperature $T_{c}$, equilibrium local magnetization $m_{\mathrm{eq}}$, and lattice constant $a$. However, the nonequilibrium part $\Delta F_{\text {ne }}$ monotonically increases with time [for $R>0$ in Eq. (4)], thus the arguments based on either maximization of entropy or minimization of free energy are not always valid [2-4]. At nonequilibrium states, the magnon backscatterings by a domain wall due to finite $R$ give rise to another effective field $\mathbf{h}_{\text {ne }}$. We note that the two effective fields $\mathbf{h}_{\mathrm{e}}$ and $\mathbf{h}_{\mathrm{ne}}$ must be additive because entropy/free energy is additive. In the following, we study the magnon transport, in particular its backscatterings by a domain wall, and predict a thermodynamic magnon recoil effect in the presence of a temperature gradient, competing with the entropic force.

The thermal properties of magnons crucially depend on their dispersion relations and lifetime. To calculate the magnon thermal conductivity $\kappa$, we consider the heat current carried by the magnon flow due to the temperature gradient $\nabla T$ in the absence of domain walls (as shown in the upper panel in Fig. 1), $\mathbf{j}_{\varepsilon}^{\mathrm{I}}=L^{-1} \sum_{k} \delta n_{k} \hbar \omega \mathbf{v}_{g}(\mathbf{k})$, where $L$ is the wire length, $\mathbf{k}$ is the magnon wave vector, $\delta n_{k}=n_{k}-\bar{n}_{k}$ is the magnon number in excess of equilibrium value $\bar{n}_{k}=1 /\left[e^{\hbar \omega(k) /\left(k_{B} T\right)}-1\right]$ being the Bose-Einstein distribution with magnon frequency $\omega(k)=$ $\varepsilon / \hbar$, and $\mathbf{v}_{g}(\mathbf{k})=\partial \omega / \partial \mathbf{k}$ is the magnon group velocity. Using the Boltzmann approach we can write a first-order expression for the excess magnon number in the steady state and in the relaxation time approximation, $\delta n_{k}=-\tau_{k}\left(\partial \bar{n}_{k} / \partial T\right) \mathbf{v}_{g} \cdot \nabla T$, where $\tau_{k}$ is the magnon relaxation time. One thus obtains the magnon thermal conductivity

$$
\kappa=\frac{1}{2 \pi} \sum_{n=1}^{N} \int_{\omega_{n}^{\min }}^{\omega_{n}^{\max }} \tau_{k} \hbar \omega\left(\partial \bar{n}_{k} / \partial T\right) v_{g} d \omega,
$$

by using the one-dimensional magnon DOS $\rho(k)=L / 2 \pi$. Here $N$ is the number of energy bands and $\omega_{n}^{\min (\max )}$ is the lowest (highest) frequency of each band $n$.

The presence of a domain wall may lead to a strong spin-wave reflection, and thus a reduction of magnon heat currents $\mathbf{j}_{\varepsilon}^{\text {II }}=(1-R) \mathbf{j}_{\varepsilon}^{\text {I. }}$. The reported mean free path of thermal magnons in insulating ferromagnets, e.g., YIG, usually is $\sim 1-100 \mu \mathrm{m}[37,38]$, which is much larger than the domain 
wall width $\delta_{D} \sim 10-100 \mathrm{~nm}$; the scattering of spin waves by the wall can thus be treated as a ballistic process, thereby conserving the total momentum. We then can derive the reflection probability $R$ of magnon heat currents by the wall via the Landauer-Büttiker formula [26,39]

$$
R(T)=\frac{\sum_{n=1}^{N} \int_{\omega_{n}^{\min }}^{\omega_{\max }^{\max }} G(\omega, T)|r(k)|^{2} d \omega}{\sum_{n=1}^{N} \int_{\omega_{n}^{\min }}^{\omega_{\max }} G(\omega, T) d \omega},
$$

where $r(k)$ is the $k$-dependent reflection coefficient of magnons by the wall and $G(\omega, T)=\hbar \omega\left(\partial \bar{n}_{k} / \partial T\right)$. Here we do not consider the modification of the magnon DOS due to the wall [26], which is relevant to reflectionless magnons treated in equilibrium thermodynamic theories $[4,40]$ but causes only negligible effects to our results here. In the momentumconserving scattering process between spin waves and the domain wall, the change rate of the linear momentum of a $\mathrm{DW}$ is $d p_{\mathrm{DW}} / d t=2 \dot{\phi} M_{s} / \gamma$ [23] which must be compensated by that from magnons (with wave vector $k$ ) $d p_{\text {magnons }} / d t=$ $\left(\delta n_{k}\right) v_{g}|r(k)|^{2} \hbar k$. Here $\phi$ is the tilted angle of the DW plane, $M_{s}$ is the saturation magnetization, and $\gamma$ is the gyromagnetic ratio. Spin-wave reflections thus lead to a precession of the domain wall plane with the angular velocity

$$
\dot{\phi}_{k}=\frac{\gamma}{2 M_{s}}\left(\delta n_{k}\right) v_{g}|r(k)|^{2} \hbar k .
$$

The equivalent magnetic field responsible for the above precession velocity is then $\mathbf{h}_{k}=\dot{\phi}_{k} / \gamma$, giving rise to an effective field along the wire axis after a summation of all states

$$
\begin{aligned}
\mathbf{h}_{\mathrm{ne}} & =L^{-1} \sum_{k} \mathbf{h}_{k} \\
& =-\frac{\nabla T}{4 \pi M_{s}} \sum_{n=1}^{N} \int_{\omega_{n}^{\min }}^{\omega_{n}^{\max }} \tau_{k}\left(\partial \bar{n}_{k} / \partial T\right) v_{g}|r(k)|^{2} \hbar k d \omega,
\end{aligned}
$$

which is the effective field or force acting on the wall due to the thermodynamic magnon recoil in a temperature gradient.

Equation (8) is quite a general formula that can be used to calculate the effective field under any magnon dispersion relations and relaxation mechanisms. Experiment data in YIG, for instance, show an acoustic branch with frequency that rises from nearly zero at the Brillouin zone center to a value at the zone boundary that varies from 6 to $9.5 \mathrm{THz}$. These values correspond to temperatures of approximately 300 and $500 \mathrm{~K}$. Since the lowest optical branch lies above the energy at zone boundaries, the calculation of the thermal properties up to room temperature can be done considering only the acoustic branch, i.e., $N=1$. At low wave numbers the dispersion relation can be approximately by a quadratic form $\omega=\omega_{1}^{\min }+J k^{2}$, where $\omega_{1}^{\min }$ is the acoustic band gap depending on materials parameters, such as the magnetic anisotropy, dipole-dipole coupling, Dzyaloshiskii-Moriya interaction, etc., and $J$ is the exchange constant. The temperature dependence of exchange constant $J$ is neglected, since it only contributes a secondary effect to the magnon recoil force. The cut-off frequency $\omega_{1}^{\max }$ thus is $\omega_{1}^{\max }=\omega_{1}^{\min }+J k_{\mathrm{m}}^{2}$ with $k_{\mathrm{m}}$ the maximum wave vector depending on the magnon propagation direction. The magnon group velocity is then $v_{g}=2 \sqrt{J\left(\omega-\omega_{1}^{\mathrm{min}}\right)}$. It has been shown that the quadratic dispersion agrees very well with the actual dispersion up to a wave vector $k=0.6 k_{\mathrm{m}}$ in YIG [41]. Under the above conditions, we obtain

$$
\mathbf{h}_{\mathrm{ne}}=-\frac{\kappa \nabla T}{2 \pi M_{s}} \frac{|\bar{r}|^{2}}{\bar{v}_{g}},
$$

with the average reflection probability

$|\bar{r}|^{2}=\int_{\omega_{1}^{\min }}^{\omega_{1}^{\max }} \tau_{k} G(\omega, T)|r(k)|^{2} d \omega / \int_{\omega_{1}^{\min }}^{\omega_{1}^{\max }} \tau_{k} G(\omega, T) d \omega$,

and the average group velocity

$$
\bar{v}_{g}=\int_{\omega_{1}^{\min }}^{\omega_{1}^{\max }} \tau_{k} v_{g} G(\omega, T) d \omega / \int_{\omega_{1}^{\min }}^{\omega_{1}^{\max }} \tau_{k} G(\omega, T) d \omega .
$$

We then obtain the DW velocity along the direction of heat currents due to their recoil effect, below Walker breakdown [9],

$$
\mathbf{v}_{\mathrm{ne}}(T)=\frac{\gamma \delta_{D}}{\alpha} \mathbf{h}_{\mathrm{ne}}=-\frac{|\bar{r}|^{2}}{\bar{v}_{g}} \frac{\gamma \delta_{D}}{\alpha} \frac{\kappa \nabla T}{2 \pi M_{s}} .
$$

So, the final DW propagation direction depends on the competition between $\mathbf{h}_{\mathrm{ne}}$ and $\mathbf{h}_{\mathrm{e}}$. The condition to observe a DW propagation toward the colder regime is therefore

$$
\kappa \frac{|\bar{r}|^{2}}{\bar{v}_{g}}>\frac{2 \pi}{\delta_{D} m_{\mathrm{eq}} a}\left(J_{0} / T_{c}\right)
$$

which requires a good heat conduction in magnetic domains (a large $\kappa$ without the domain wall), a strong magnon backscattering (a large $|\bar{r}|^{2}$ ), a slow magnon group velocity (a small $\bar{v}_{g}$ ), and a broad domain wall (a large $\delta_{D}$ ). The forces induced by nonequilibrium thermal fluctuations under temperature gradients cause a Brownian motion $[42,43]$ of the domain wall and could be another reason to push its propagation toward the colder region [44]. However, it is still an open question how valid the classical fluctuation-dissipation theorem for equilibrium states is [45], particularly when it is applied to nonequilibrium steady states in the presence of temperature gradients [46-49], that are beyond our formalism.

In order to evaluate the parameters in criterion (13), we now can make either of two plausible assumptions about the behavior of $\tau_{k}$. Model I: If one considers that the relaxation time $\tau_{k}$ is independent of both the wave number and the temperature (the simple average-lifetime model) [50] and takes $\tau_{k}=\bar{\tau}_{k}$, we obtain $\kappa \propto \bar{\tau}_{k}$. Model II: If we consider the Gilbert damping but neglect higher order processes such as magnon-magnon and magnon-phonon interactions, the relaxation time is then $\tau_{k}=1 /(2 \alpha \omega)$ with Gilbert damping constant $\alpha$ [31]. We thus have $\kappa \propto 1 / \alpha$.

It has been shown that both dipole-dipole [23] and Dzyaloshiskii-Moriya [29] interactions can result in strong magnon reflections in the presence of a domain wall in ferromagnets. A precessing domain wall in antiferromagnets can also leads to significant magnon reflections [28,51]. A common feature of the reflection probability function $|r[k(\omega)]|^{2}$ is the sharp transition from 1 at lower frequencies to 0 at higher frequencies [23,24,51], satisfying ansatz $|r[k(\omega)]|^{2}=$ $\left|w\left[-\left(\omega-\omega_{c}\right) / \Delta \omega\right]\right|^{2}$, with the transition frequency $\omega_{c}$ and the spectrum width $\Delta \omega$. Function $|w|^{2}$ reduces to 1 for $\omega \ll \omega_{c}$, and 0 for $\omega \gg \omega_{c}$. The form of the function $w$ depends 
on material parameters such as the domain wall width [26], the Dzyaloshiskii-Moriya interaction strength [29], etc., and scattering details such as the incident angle of magnons [23]. However, for a very narrow spectrum $(\Delta \omega \ll 1)$ which is often the case $[23,51]$, it can be approximately described by $|w|^{2} \approx s\left[-\left(\omega-\omega_{c}\right)\right]$ with the Heaviside step function $s(x)$. It indicates that magnons are completely reflected by the wall when their frequencies are lower than $\omega_{c}$, while they essentially pass through the domain wall without any reflection above $\omega_{c}$. By denoting $A=\hbar \omega_{1}^{\min } / k_{B}, B=\hbar \omega_{1}^{\max } / k_{B}, x_{a}=$ $A / T$, and $x_{c}=C / T$ with $C=\hbar \omega_{c} / k_{B}$, Eqs. (5), (10), and (11) can be calculated analytically and yield

$$
\begin{gathered}
\kappa=\frac{\bar{\tau}_{k}}{\pi} \sqrt{\frac{J k_{B}^{5} T^{3}}{\hbar^{3}}} \int_{A / T}^{B / T} \frac{\sqrt{x-x_{a}} x^{2} e^{x}}{\left(e^{x}-1\right)^{2}} d x, \\
\bar{v}_{g}=2 \sqrt{\frac{J k_{B} T}{\hbar}} \frac{\int_{A / T}^{B / T} \frac{\sqrt{x-x_{a}} x^{2} e^{x}}{\left(e^{x}-1\right)^{2}} d x}{f(A / T)-f(B / T)}, \\
|\bar{r}|^{2}=R=\frac{\int_{A / T}^{B / T} s\left[-\left(x-x_{c}\right)\right] \frac{x^{2} e^{x}}{\left(e^{x}-1\right)^{2}} d x}{f(A / T)-f(B / T)},
\end{gathered}
$$

with [52]

$$
f(x)=-2 x \ln \left(1-e^{-x}\right)+\frac{x^{2}}{e^{x}-1}+2 \sum_{p=1}^{\infty} \frac{e^{-p x}}{p^{2}},
$$

in model I $\left(\tau_{k}=\bar{\tau}_{k}\right)$, and

$$
\begin{gathered}
\kappa=\frac{1}{2 \pi \alpha} \sqrt{\frac{J k_{B}^{3} T}{\hbar}} \int_{A / T}^{B / T} \frac{\sqrt{x-x_{a}} x e^{x}}{\left(e^{x}-1\right)^{2}} d x, \\
\bar{v}_{g}=2 \sqrt{\frac{J k_{B} T}{\hbar}} \frac{\int_{A / T}^{B / T} \frac{\sqrt{x-x_{a}} x e^{x}}{\left(e^{x}-1\right)^{2}} d x}{g(A / T)-g(B / T)}, \\
|\bar{r}|^{2}=\frac{\int_{A / T}^{B / T} s\left[\left(-x-x_{c}\right)\right] \frac{x e^{x}}{\left(e^{x}-1\right)^{2}} d x}{g(A / T)-g(B / T)},
\end{gathered}
$$

with [52]

$$
g(x)=-\ln \left(1-e^{-x}\right)+\frac{x}{e^{x}-1}
$$

in model II $\left[\tau_{k}=1 /(2 \alpha \omega)\right]$.

The following three cases are of potential interest. (i) For a low transition frequency $\left(\omega_{c}<\omega_{1}^{\mathrm{min}}\right)$, there is no reflection, and Eqs. (16) and (20) yield $|\bar{r}|^{2}=0$. (ii) For an intermediate transition frequency $\left(\omega_{1}^{\min }<\omega_{c}<\omega_{1}^{\max }\right)$, Eqs. (16) and (20) reduce to $|\bar{r}|^{2}=[f(A / T)-f(C / T)] /[f(A / T)-f(B / T)]$ and $[g(A / T)-g(C / T)] /[g(A / T)-g(B / T)]$, respectively. (iii) For a high transition frequency $\left(\omega_{c}>\omega_{1}^{\max }\right)$, all magnons are reflected by the wall. Thus, Eqs. (16) and (20) reduce to $|\bar{r}|^{2}=1$.

Figure 2(a) shows the temperature dependence of the magnon thermal conductivity $\kappa$ in both models I and II. It increases as the temperature is elevated in both cases. In Fig. 2(b) we calculate the average group velocity $\bar{v}_{g}$. It monotonically increases with the temperature and saturates at high temperatures. The temperature dependence of $|\bar{r}|^{2}$ for different transition temperatures $C$ is shown in Fig. 2(c), with a monotonically decreasing manner. It is because higher temperatures make more magnons populate higher energy lev-
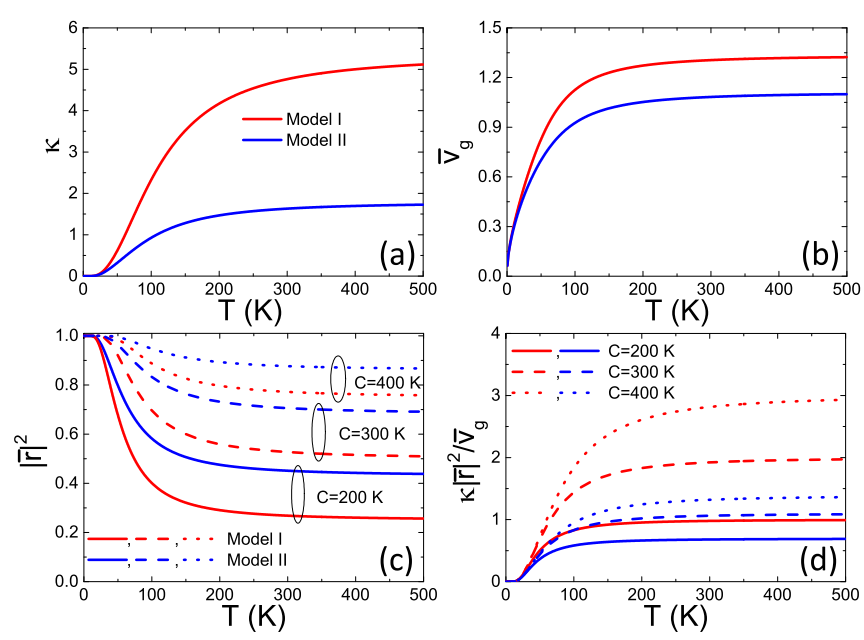

FIG. 2. (Color online) (a) Temperature dependence of the magnon heat conductivity $\kappa$ normalized by $\bar{\tau}_{k} \sqrt{J k_{B}^{5} A^{3} / \pi^{3} \hbar^{3}}$ in model I, and by $(2 \pi \alpha)^{-1} \sqrt{J k_{B}^{3} A / \hbar}$ in model II, respectively. (b) Average group velocity $\bar{v}_{g}$ as a function of temperature $T$, in units of $2 \sqrt{J k_{B} A / \hbar}$ in two models. (c) Temperature dependence of average reflection probability $|\bar{r}|^{2}$ for different transition temperatures $C$. (d) Parameter $\kappa|\bar{r}|^{2} / \bar{v}_{g}$ as a function of temperature for different $C$, with units of $\bar{\tau}_{k} k_{B}^{2} A /(2 \pi \hbar)$ in model I and of $k_{B} /(4 \pi \alpha)$ in model II, respectively. In the calculations, we use $A=100 \mathrm{~K}$ and $B=500 \mathrm{~K}$. All temperatures are below $T_{c}$.

els, which subsequently leads to a smaller magnon reflection. We also observe that a higher transition temperature leads to a stronger magnon reflection. Figure 2(d) demonstrates a monotonically increasing dependence on the temperature of parameter $\kappa|\bar{r}|^{2} / \bar{v}_{g}$ in both models. As shown in Eq. (13) with quadratic magnon dispersion relations, parameter $\kappa|\bar{r}|^{2} / \bar{v}_{g}$, which is independent of exchange constant $J$, is crucial to determine if the domain wall can move toward the colder region. According to our calculations, this condition should be reasonably satisfied at elevated temperatures $(T \gtrsim 400 \mathrm{~K})$ with a small magnon damping $\left(\bar{\tau}_{k} \gtrsim 1\right.$ ns or $\left.\alpha \lesssim 10^{-4}\right)$ for a broad domain wall $\left(\delta_{D} \gtrsim 100 \mathrm{~nm}\right)$ in a weak ferromagnet $\left(J_{0} / a \lesssim 3 \times 10^{-12} \mathrm{~J} / \mathrm{m}\right)$ under any temperature gradient that can overcome the pinning force produced by defects or impurities. Other relaxation models considering three- and four-magnon scattering processes are expected not to modify our conclusions significantly.

To summarize, we predict a thermodynamic magnon recoil effect for domain wall motion under temperature gradients. We correct the previous thermodynamic theories by including a heat current term for entropy and/or free-energy generations, which is always present in nonequilibrium steady states in the presence of a temperature gradient. The heat current gets modulations by the DW with momentum-conserving backscatterings. It then leads to a recoil force on the wall, which competes with the previously identified entropic force. Our theory thereby closes the inconsistency between macroscopic and microscopic theories for the domain wall motion, and we propose experiments to test it. We also expect the similar thermodynamic magnon recoil effect to play an important role in other magnetic structures, e.g., magnetic vortices, bubbles, or skyrmions, and other materials like antiferromagnets or multiferroics. 
Note added. Recently, we became aware of one recent report [53] on a DW thermophoresis in antiferromagnets using the classical fluctuation-dissipation relation without considering any magnon backscattering. Our results should also be applicable to their work.
We thank Se Kwon Kim and Gerrit Bauer for useful discussions. This work is supported by DFG Priority Programme 1538 "Spin-Caloric Transport" and the Alexander Von Humboldt Foundation. Y.C. acknowledges the financial support from European Union Seventh Framework Programme "SpinIcur" under Grant No. FP7-People-2012-ITN-316657.
[1] G. E. W. Bauer, E. Saitoh, and B. J. van Wees, Nat. Mater. 11, 391 (2012).

[2] D. Hinzke and U. Nowak, Phys. Rev. Lett. 107, 027205 (2011).

[3] F. Schlickeiser, U. Ritzmann, D. Hinzke, and U. Nowak, Phys. Rev. Lett. 113, 097201 (2014).

[4] X. S. Wang and X. R. Wang, Phys. Rev. B 90, 014414 (2014).

[5] A. A. Kovalev and Y. Tserkovnyak, Europhys. Lett. 97, 67002 (2012).

[6] W. Jiang, P. Upadhyaya, Y. B. Fan, J. Zhao, M. S. Wang, L. T. Chang, M. R. Lang, K. L. Wong, M. Lewis, Y. T. Lin, J. S. Tang, S. Cherepov, X. Z. Zhou, Y. Tserkovnyak, R. N. Schwartz, and K. L. Wang, Phys. Rev. Lett. 110, 177202 (2013).

[7] D. A. Allwood, G. Xiong, C. C. Faulkner, D. Atkinson, D. Petit, and R. P. Cowburn, Science 309, 1688 (2005).

[8] S. S. P. Parkin, M. Hayashi, and L. Thomas, Science 320, 190 (2008).

[9] N. L. Schryer and L. R. Walker, J. Appl. Phys. 45, 5406 (1974).

[10] D. Atkinson, D. A. Allwood, G. Xiong, M. D. Cooke, C. C. Faulkner, and R. P. Cowburn, Nat. Mater. 2, 85 (2003).

[11] G. S. D. Beach, C. Nistor, C. Knutson, M. Tsoi, and J. L. Erskine, Nat. Mater. 4, 741 (2005).

[12] M. Hayashi, L. Thomas, Ya. B. Bazaliy, C. Rettner, R. Moriya, X. Jiang, and S. S. P. Parkin, Phys. Rev. Lett. 96, 197207 (2006).

[13] L. Berger, Phys. Rev. B 54, 9353 (1996); J. Slonczewski, J. Magn. Magn. Mater. 159, L1 (1996).

[14] S. Zhang and Z. Li, Phys. Rev. Lett. 93, 127204 (2004).

[15] G. Tatara and H. Kohno, Phys. Rev. Lett. 92, 086601 (2004).

[16] I. M. Miron, K. Garello, G. Gaudin, P. J. Zermatten, M. V. Costache, S. Auffret, S. Bandiera, B. Rodmacq, A. Schuhl, and P. Gambardella, Nature (London) 476, 189 (2011).

[17] L. Liu, C. F. Pai, Y. Li, H. W. Tseng, D. C. Ralph, and R. A. Buhrman, Science 336, 555 (2012).

[18] A. V. Khvalkovskiy, V. Cros, D. Apalkov, V. Nikitin, M. Krounbi, K. A. Zvezdin, A. Anane, J. Grollier, and A. Fert, Phys. Rev. B 87, 020402(R) (2013).

[19] P. P. J. Haazen, E. Murè, J. H. Franken, R. Lavrijsen, H. J. M. Swagten, and B. Koopmans, Nat. Mater. 12, 299 (2013).

[20] S. Emori, U. Bauer, S.-M. Ahn, E. Martinez, and G. S. D. Beach, Nat. Mater. 12, 611 (2013).

[21] M. Hatami, G. E. W. Bauer, Q. Zhang, and P. J. Kelly, Phys. Rev. Lett. 99, 066603 (2007).

[22] P. Yan, X. S. Wang, and X. R. Wang, Phys. Rev. Lett. 107, 177207 (2011).

[23] P. Yan, A. Kamra, Y. Cao, and G. E. W. Bauer, Phys. Rev. B 88, 144413 (2013).

[24] X. G. Wang, G. H. Guo, Y. Z. Nie, G. F. Zhang, and Z. X. Li, Phys. Rev. B 86, 054445 (2012).
[25] J. S. Kim, M. Stärk, M. Kläui, J. Yoon, C. Y. You, L. Lopez-Diaz, and E. Martinez, Phys. Rev. B 85, 174428 (2012).

[26] P. Yan and G. E. W. Bauer, Phys. Rev. Lett. 109, 087202 (2012).

[27] E. G. Tveten, A. Qaiumzadeh, and A. Brataas, Phys. Rev. Lett. 112, 147204 (2014).

[28] S. K. Kim, Y. Tserkovnyak, and O. Tchernyshyov, Phys. Rev. B 90, 104406 (2014).

[29] W. Wang, M. Albert, M. Beg, M.-A. Bisotti, D. Chernyshenko, D. Cortés-Ortuño, I. Hawke, and H. Fangohr, Phys. Rev. Lett. 114, 087203 (2015).

[30] W. F. Brown, Phys. Rev. 130, 1677 (1963).

[31] T. L. Gilbert, IEEE Trans. Magn. 40, 3443 (2004).

[32] R. Kubo and N. Hashitsume, Prog. Theor. Phys. Suppl. 46, 210 (1970).

[33] J. L. García-Palacios and F. J. Lázaro, Phys. Rev. B 58, 14937 (1998).

[34] J. M. Luttinger, Phys. Rev. 135, A1505 (1964).

[35] G. Tatara, Phys. Rev. Lett. 114, 196601 (2015).

[36] L. D. Landau, E. M. Lifshitz, and L. P. Pitaevskii, Electrodynamics of Continuous Media (Pergamon, New York, 1984).

[37] U. Ritzmann, D. Hinzke, and U. Nowak, Phys. Rev. B 89, 024409 (2014).

[38] S. R. Boona and J. P. Heremans, Phys. Rev. B 90, 064421 (2014).

[39] F. Meier and D. Loss, Phys. Rev. Lett. 90, 167204 (2003).

[40] B. Sangiorgio, T. C. T. Michaels, D. Pescia, and A. Vindigni, Phys. Rev. B 89, 014429 (2014).

[41] S. M. Rezende and J. C. López Ortiz, Phys. Rev. B 91, 104416 (2015).

[42] A. Najafi and R. Golestanian, Europhys. Lett. 68, 776 (2004).

[43] L. Kong and J. Zang, Phys. Rev. Lett. 111, 067203 (2013).

[44] S. K. Kim (private communication).

[45] C. H. Woo, H. Wen, A. A. Semenov, S. L. Dudarev, and P.-W. Ma, Phys. Rev. B 91, 104306 (2015).

[46] R. Chetrite, G. Falkovich, and K. Gawedzki, J. Stat. Mech. (2008) P08005.

[47] J. R. Gomez-Solano, A. Petrosyan, S. Ciliberto, R. Chetrite, and K. Gawedzki, Phys. Rev. Lett. 103, 040601 (2009).

[48] J. Prost, J.-F. Joanny, and J. M. R. Parrondo, Phys. Rev. Lett. 103, 090601 (2009).

[49] U. Seifert and T. Speck, Europhys. Lett. 89, 10007 (2010).

[50] W. B. Yelon and L. Berger, Phys. Rev. B 6, 1974 (1972).

[51] M. M. Bogdan and O. V. Charkina, Low Temp. Phys. 40, 84 (2014).

[52] M. Abramowitz and I. A. Stegun, Handbook of Mathematical Functions (Dover, New York, 2012).

[53] S. K. Kim, O. Tchernyshyov, and Y. Tserkovnyak, Phys. Rev. B 92, 020402(R) (2015). 